\title{
Usual limit shadowable homoclinic classes of generic diffeomorphisms
}

\author{
Manseob Lee
}

Correspondence: Imsds@mokwon. ac.kr

Department of Mathematics, Mokwon University, Daejeon, 302729, Korea

\section{Abstract}

We show that for $C^{1}$-generic $f$, a locally maximal homoclinic class is usual limit shadowable if and only if the homoclinic class is hyperbolic.

Mathematics Subject Classification 2010: 37C20; 37C40; 37C50; 34D05.

Keywords: usual limit shadowing, hyperbolic, homoclinic class, $C^{1}$-generic

\section{Introduction}

Let $M$ be a closed $C^{\infty} n$-dimensional manifold, and let $\operatorname{Diff}(M)$ be the space of diffeomorphisms of $M$ endowed with the $C^{1}$-topology. Denote by $d$ the distance on $M$ induced from a Riemannian metric $\|\cdot\|$ on the tangent bundle TM. Let $f \in \operatorname{Diff}(M)$. For $\delta>0$, a sequence of points $\left\{x_{i}\right\}_{i=a}^{b}(-\infty \leq a<b \leq \infty)$ in $M$ is called a $\delta$-pseudo orbit of $f$ if $d\left(f\left(x_{i}\right), x_{i+1}\right)<\delta$ for all $a \leq i \leq b-1$. For given $x, y \in M$, we write $x \leadsto y$ if for any $\delta>0$, there is a $\delta$-pseudo orbit $\left\{x_{i}\right\}_{i=a}^{b}(a<b)$ of $f$ such that $x_{a}=x$ and $x_{b}=y$. Let $\Lambda \subset M$ be a closed $f$-invariant set. We say that $f$ has the shadowing property on $\Lambda$ if for every $>0$ there is $\delta>0$ such that for any $\delta$-pseudo orbit $\left\{x_{i}\right\}_{i=a}^{b} \subset \Lambda$ of $f(-\infty \leq a$ $<b \leq \infty)$, there is a point $y \in M$ such that $d\left(f^{\prime}(y), x_{i}\right)<\epsilon$ for all $a \leq i \leq b-1$. The notion of pseudo orbits often appears in several methods of the modern theory of dynamical system [1]. Moreover, the shadowing property usually plays an important role in the investigation of stability theory and ergodic theory. Recently, Abdenur and Dìa [2] given a problem which $C^{1}$-generically, a diffeomorphism has the shadowing property if and only if it is hyperbolic. Therefore, we study the some kinds of the shadowing property (usual limit shadowing property) and homoclinic classes. As to the research on the usual limit shadowing property, there exist $[1,3,4]$. We say that $f$ has the usual limit shadowing property on $\Lambda$ if for any sequence $\xi=\left\{x_{i}\right\}_{i \in \mathbb{N}} \subset \Lambda$ such that $d\left(f\left(x_{i}\right), x_{i+1}\right) \rightarrow$ 0 as $i \rightarrow \pm \infty$, there exists a point $y \in M$ such that $d\left(f(y), x_{i}\right) \rightarrow 0$ as $i \rightarrow \pm \infty$. In this case, the sequence $\xi$ is called a limit pseudo orbit of $f$. Note that the usual limit shadowing property is not equivalent to the shadowing property (see, [1]). We say that $\Lambda$ is locally maximal if there is a compact neighborhood $U$ of $\Lambda$ such that

$$
\bigcap_{n \in \mathbb{Z}} f^{n}(U)=\Lambda \text {. }
$$

We say that $\Lambda$ is hyperbolic if the tangent bundle $T_{\Lambda} M$ has a $D$-invariant splitting $E^{s}$ $\oplus E^{u}$ and there exist constants $C>0$ and $0<\lambda<1$ such that

(c) 2012 Lee; licensee Springer. This is an Open Access article distributed under the terms of the Creative Commons Attribution License (http://creativecommons.org/licenses/by/2.0), which permits unrestricted use, distribution, and reproduction in any medium, provided the original work is properly cited. 


$$
\left\|\left.D_{x} f^{n}\right|_{E_{x}^{s}}\right\| \leq C \lambda^{n} \text { and }\left\|\left.D_{x} f^{-n}\right|_{E_{x}^{u}}\right\| \leq C \lambda^{n}
$$

for all $x \in \Lambda$ and $n \geq 0$. Moreover, we say that $\Lambda$ admits a dominated splitting if the tangent bundle $T_{\Lambda} M$ has a continuous $D f$-invariant splitting $E \oplus F$ and there exist constants $C>0$ and $0<\lambda<1$ such that

$$
\left\|\left.D_{x} f^{n}\right|_{E(x)}\right\| \cdot\left\|\left.D_{x} f^{-n}\right|_{F\left(f^{n}(x)\right)}\right\| \leq C \lambda^{n}
$$

for all $x \in \Lambda$ and $n \geq 0$.

Let $p \in P(f)$ be a hyperbolic saddle with period $\pi(p)>0$. Then there are the local stable manifold $W_{\epsilon}^{s}(p)$ and the unstable manifold $W_{\epsilon}^{u}(p)$ of $p$ for some $\epsilon=\epsilon(p)>0$. It is easily seen that if $d\left(\rho^{n}(x), f^{n}(p)\right) \leq \epsilon$, for all $n \geq 0$ then $x \in W_{\epsilon}^{s}(p)$, and if $d\left(\rho^{n}(x), f^{n}\right.$ $(p)) \leq \epsilon$ for all $n \leq 0$ then $x \in W_{\epsilon}^{u}(p)$. The stable manifold $W^{s}(p)$ and the unstable manifold $W^{\mu}(p)$ defined as followings. It is well known that if $p$ is a hyperbolic periodic point of $f$ with period $k$ then the sets

$$
\begin{aligned}
& W^{s}(p)=\left\{x \in M: f^{k n}(x) \rightarrow p \text { as } n \rightarrow \infty\right\} \text { and } \\
& W^{u}(p)=\left\{x \in M: f^{-k n}(x) \rightarrow p \text { as } n \rightarrow \infty\right\}
\end{aligned}
$$

are $C^{1}$-injectively immersed submanifolds of $M$.

A point $x \in W^{\mathcal{S}}(p) \cap W^{\mu}(p)$ is called a homoclinic point of $f$ associated to $p$, and it is said to be a transversal homoclinic point of $f$ if the above intersection is transversal at $x$; i.e., $x \in W^{s}(p) \pitchfork W^{\mu}(p)$. The closure of the transversal homoclinic points of $f$ associated to $p$ is called the transversal homoclinic class of $f$ associated to $p$, and it is denoted by $H_{f}(p)$. Let $q$ be a hyperbolic periodic point of $f$. We say that $p$ and $q$ are homoclinically related, and write $p \sim q$ if

$$
W^{s}(p) \pitchfork W^{u}(q) \neq \emptyset \quad \text { and } \quad W^{u}(p) \pitchfork W^{s}(q) \neq \emptyset .
$$

It is clear that if $p \sim q$ then index $(p)=\operatorname{index}(q)$; i.e., $\operatorname{dim} W^{s}(p)=\operatorname{dim} W^{s}(q)$. By the Smale's transverse homoclinic point theorem, $H_{f}(p)$ coincides with the closure of the set of hyperbolic periodic points $q$ of $f$ such that $p \sim q$. The homoclinic class is a transitive set, compact and $f$-invariant set. Homoclinic classes are the natural candidates to replace hyperbolic basic sets, for instance, if $f$ is Axiom A, then Smale's spectral decomposition theorem says that the non-wandering set can be decomposed into finitely many basic sets, and each basic set is a homoclinic class.

We say that a subset $\mathcal{G} \subset \operatorname{Diff}(M)$ is residual if $\mathcal{G}$ contains the intersection of a countable family of open and dense subsets of $\operatorname{Diff}(M)$; in this case $\mathcal{G}$ is dense in Diff $(M)$. A property "P" is said to be $\left(C^{1}\right)$-generic if " $\mathrm{P}$ " holds for all diffeomorphisms which belong to some residual subset of $\operatorname{Diff}(M)$. We use the terminology "for $C^{1}$ generic $f^{\prime \prime}$ to express "there is a residual subset $\mathcal{G} \subset \operatorname{Diff}(M)$ such that for any $f \in \mathcal{G}$...".

We prove that $C^{1}$-generically, $\Lambda$ is a locally maximal homoclinic class containing hyperbolic periodic point $p$, and $f$ has the usual limit shadowing property if and only if the homoclinic class is hyperbolic.

Very recently, Lee and Wen [5] showed that $C^{1}$ generically, a locally maximal chain transitive sets is shadowable if and only if it is hyperbolic. From the above facts, we study relations between the usual limit shadowing property and hyperbolic. From now, we only consider the homoclinic class containing a hyperbolic periodic point $p$ which 
is saddle because, if the point $p$ is sinks or sources then they are one orbits. In this article, the following is the main result.

Theorem 1.1 For $C^{1}$ generic $f$, a locally maximal homoclinic class $H_{f}(p)$ is usual limit shadowable if and only if the homoclinic class $H_{f}(p)$ is hyperbolic.

\section{Proof of Theorem1.1}

Let $M$ be as before, and let $f \in \operatorname{Diff}(M)$. Denote by $P(f)$ the set of periodic points of $f$. Let $p$ be a hyperbolic periodic point of $f$.

Proposition 2.1 There is a residual set $\mathcal{G}^{\prime} \subset \operatorname{Diff}(M)$ such that for any $f \in \mathcal{G}^{\prime}$, if a locally maximal homoclinic class $H_{f}(p)$ is usual limit shadowable then for any hyperbolic $q \in H_{f}(p) \cap P(f)$,

$$
\operatorname{index}(p)=\operatorname{index}(q) \text {, }
$$

where index $(p)=\operatorname{dim} W^{S}(p)$.

To prove Proposition 2.1, we need the following lemmas.

Lemma 2.2 Let $H_{f}(p)$ be a homoclinic class of $p$. Suppose that $f$ has the usual limit shadowing property on $H_{f}(p)$. Then for any $q \subset H_{f}(p) \cap P_{h}(f)$,

$$
W^{s}(p) \cap W^{u}(q) \neq \emptyset, \quad \text { and } \quad W^{u}(p) \cap W^{s}(q) \neq \emptyset,
$$

where $P_{h}(f)$ is the set of hyperbolic periodic points of $f$.

Proof. Let $q \in H_{f}(p)$ be a hyperbolic periodic point of $f$. Suppose that $f$ has the usual limit shadowing property on $H_{f}(p)$. To simplify, assume that $f(p)=p$ and $f(q)=q$. Since $q \in H_{f}(p)$ and $H_{f}(p)=\overline{\{q \in P(f): q \sim p\}}$, for any small $\eta>0$, we can take $x \in$ $H_{f}(p)$ and $k>0$ such that $x \sim p, d\left(f^{k}(x), q\right)<\eta$ and $d\left(f^{k}(x), p\right)<\eta$.

Then we can construct a limit pseudo orbit $\xi$ as follows.

$$
\text { Put } f^{k-i}(q)=x_{-k-i}, f^{k+i}(p)=x_{k+i} \text { for } i \geq 0 \text { and } f^{i}(x)=x_{i}, f^{i}(x)=x_{-i} \text { for } 0 \leq i \leq k-1 \text {. }
$$

Then

$$
\begin{aligned}
\xi & =\left\{\ldots, q, q, f^{-k+1}(x), \ldots, f^{-1}(x), x, f(x), \ldots, f^{k-1}(x), p, p, \ldots\right\} \\
& =\left\{\ldots, x_{-k-1}, x_{-k}, x_{-k+1}, \ldots, x_{-1}, x_{0}(=x), x_{1}, \ldots, x_{k-1}, x_{k}, \ldots\right\} .
\end{aligned}
$$

It is clearly, $\xi \subset H_{f}(p)$.

Since $f$ has the usual limit shadowing property on $H_{f}(p)$, we can find a point $y \in M$ such that $d\left(\dot{f}(y), x_{i}\right) \rightarrow 0$ as $i \rightarrow \pm \infty$.

Since $x_{-k-i}=q$ and $x_{k+i}=p$ for $i \geq 0, d\left(f^{\dot{f}}(y), p\right)<\epsilon$ for all $i \geq k$, and $d\left(f^{\dot{f}}(y), q\right)<\epsilon$ for all $i \leq-k$.

Therefore, $\mathcal{O}_{f}(\gamma) \cap W^{s}(p) \neq \emptyset$ and $\mathcal{O}_{f}(\gamma) \cap W^{u}(q) \neq \emptyset$. Thus, one can get

$$
W^{u}(p) \cap W^{s}(q) \neq \emptyset .
$$

Other cases is similar.

To prove Proposition 2.1, we need the following fact which is well-known KupkaSmale Theorem.

Lemma 2.3 There is a residual set $\mathcal{G}_{1} \subset \operatorname{Diff}(M)$ such that for any $f \in \mathcal{G}_{1}$, every periodic point of $f$ is hyperbolic, and the stable manifolds and the unstable manifolds of periodic points are all transverse. 
Proof of Proposition 2.1. Let $f \in \mathcal{G}^{\prime}=\mathcal{G}_{1}$, and let $q$ be a hyperbolic periodic point in $H_{f}(p)$. Suppose that $f$ has the usual limit shadowing property on $H_{f}(p)$. Then by Lemmas 2.2 and 2.3, $W^{s}(p) \pitchfork W^{u}(q) \neq \emptyset$, and $W^{u}(p) \pitchfork W^{s}(q) \neq \emptyset$. Thus index $(p)=$ index $(q)$. Therefore, $q \sim p$.

Denote by $\mathcal{F}(M)$ the set of $f \in \operatorname{Diff}(M)$ such that there is a $C^{1}$ neighborhood $\mathcal{U}(f)$ of $f$ such that for any $g \in \mathcal{U}(f)$ and every $p \in P(g)$ is hyperbolic. In [6], Hayashi proved that $f \in \mathcal{F}(M)$ if and only if $f$ satisfies both Axiom A and no-cycle condition. From the above facts, we show the following.

Proposition 2.4 There exists a residual set $\mathcal{G}^{\prime \prime} \subset \operatorname{Diff}(M)$ such that for any $f \in \mathcal{G}^{\prime \prime}$, if $f$ has the usual limit shadowing property on a locally maximal homoclinic class $H_{f}(p)$ then $f \in \mathcal{F}(M)$.

The following lemma was proved by Lee [7], here we will give sketch of the proof.

Lemma 2.5 There exists a residual set $\mathcal{G}_{2} \subset \operatorname{Diff}(M)$ such that for any $f \in \mathcal{G}_{2}$, we have the following property. For any $C^{1}$-neighborhood $\mathcal{U}(f)$ of $f$ there is $g \in \mathcal{U}(f)$ such that $g$ has two distinct hyperbolic periodic points $p_{g}, q_{g} \in P(g)$ with different indices then $f$ has two distinct hyperbolic periodic points $p, q \in P(f)$ with different indices.

Proof. Take a countable basis $\mathcal{B}=\left\{U_{n}\right\}_{n \in \mathbb{N}}$ of $M$. For each $U_{n} \in \mathcal{B}$, we defined by $\mathcal{H}_{n}$ the set of all diffeomorphisms $f$ such that $f$ has a $C^{1}$-neighborhood $\mathcal{U}(f)$ of $f$ with the following properties: for any $g \in \mathcal{U}(f)$, there are $p_{g}, q_{g} \in U_{n}$, distinct periodic hyperbolic points of $g$ with different indices. Then it is clear that $\mathcal{H}_{n}$ is open in Diff $(M)$ for each $n \in \mathbb{N}$. Let

$$
\mathcal{N}_{n}=\operatorname{Diff}(M)-\overline{\mathcal{H}_{n}} .
$$

Then $\mathcal{H}_{n} \cup \mathcal{N}_{n}$ is an open and dense subset of $\operatorname{Diff}(M)$. Put

$$
\mathcal{R}_{n}=\bigcap_{n \in \mathbb{N}} \mathcal{H}_{n} \cup \mathcal{N}_{n}
$$

is a residual subset of $\operatorname{Diff}(M)$. Then

$$
\mathcal{G}_{2}=\bigcap_{n \in \mathbb{N}} \mathcal{R}_{n}
$$

is also a residual subset of $\operatorname{Diff}(M)$. Let $f \in \mathcal{G}_{2}$. Suppose that for any $C^{1}$-neighborhood $\mathcal{U}(f)$ of $f$, there exist $g \in \mathcal{U}(f)$ and $U_{n} \in \mathcal{B}$ such that $p_{g}$ and $q_{g}$ distinct hyperbolic periodic points of $g$ with Different indices which are elements in $U_{n}$. Then $f \in \overline{\mathcal{H}_{n}}$. Since $f \in \mathcal{G}_{2}$ we know that $f \in \mathcal{H}_{n}$. Thus there are $p, q$ distinct hyperbolic periodic points of $f$ with different indices.

Now, we introduce the notion of the weak eigenvalue (see [8]). Let $p$ be a periodic point of $f$. For $0<\delta<1$, we say $p$ has a $\delta$-weak eigenvalue if $D_{\mathrm{p}} \mathrm{f}^{\pi(p)}$ has an eigenvalue $\lambda$ such that $(1-\delta)^{\pi(p)}<|\lambda|<(1+\delta)^{\pi(p)}$. Note that if $p$ is a hyperbolic periodic point of $f$ then $p$ does not have the $\delta$-weak eigenvalue. Because if $p$ has a $\delta$-weak eigenvalue then by Franks' Lemma, there exist a diffeomorpshism $g C^{1}$-nearby $f$ such that the periodic point $p_{g}$ has an eigenvalue, say $\lambda$, and $|\lambda|=1$. Thus this is a contradiction by the stability theorem.

Remark 2.6 [8, Lemma 2.1(2)] There exists a residual set $\mathcal{G}_{3} \subset \operatorname{Diff}(M)$ such that if $f \in \mathcal{G}_{3}$ then for any $\delta>0$, if for every neighborhood $\mathcal{U}(f)$ of $f$ there exist $g \in \mathcal{U}(f)$ 
which has a periodic point $q \sim p_{g}$ with $\delta$-weak eigenvalue, then $f$ has a periodic point $\gamma$ $\sim p$ with $2 \delta$-weak eigenvalue.

Lemma 2.7 [[9], Lemma 2.4] Let $\Lambda$ be locally maximal in $\mathcal{U}$, and let $\mathcal{U}(f)$ be a $C^{1}$ neighborhood of $f$. If for any $g \in \mathcal{U}(f), p \in \Lambda_{g}(U) \cap P(g)$ is not hyperbolic, then there is $g_{1} \in \mathcal{U}(f)$ possessing hyperbolic periodic points $q_{1}$ and $q_{2}$ in $\Lambda_{g 1}(U)$ with different indices, where $\Lambda_{g_{1}}(U)=\cap_{n \in \mathbb{Z}} g_{1}^{n}(U)$.

Lemma 2.8 There exists a residual set $\mathcal{G}_{4} \subset \operatorname{Diff}(M)$ such that if $f \in \mathcal{G}_{4}$, and $H_{f}(p)$ is a locally maximal homoclinic class of $p$ which satisfies the usual limit shadowing property, then there exists $\delta>0$ such that no point in $H_{f}(p)$ has a $\delta$-weak eigenvalue.

Proof. Let $\mathcal{G}_{4}=\mathcal{G}_{1} \cap \mathcal{G}_{2} \cap \mathcal{G}_{3}$. Suppose that $f$ has the usual limit shadowing property on a locally maximal homoclinic class $H_{f}(p)$. We will derive a contradiction. Let $f \in \mathcal{G}_{4}$, and $\mathcal{U}(f)$ be a $C^{1}$-neighborhood of $f$. Suppose that for $n \in \mathbb{N}$, there is $q_{n} \in H_{f}$ $(p) \cap P(f)$ such that $q_{n}$ has a $1 / n$-weak eigenvalue. By Franks' Lemma and Lemma 2.7, there exists $g \in \mathcal{U}(f)$ such that $g$ has two distinct periodic orbits $p_{g}$ and $q_{g} \in \Lambda_{g}(U)=\cap_{n \in \mathbb{Z}} g^{n}(U)$ with different indices. Since $f \in \mathcal{G}_{4}, f$ has two different periodic orbit $p$ and $q$ in $H_{f}(p)$ with different indices. Since $f$ has the the usual limit shadowing property on $H_{f}(p)$ and $f$ is a Kupka-Smale Diffeomorphism, this is a contradiction.

Proof of Proposition 2.4. Let $f \in \mathcal{G}^{\prime \prime}=\mathcal{G}_{4}$ and let $\mathcal{U}(f)$ be a $C^{1}$-neighborhood of $f$. Suppose that $f$ has the usual limit shadowing property on a locally maximal homoclinic class $H_{f}(p)$. The proof is by contradiction. Suppose that $f \notin \mathcal{F}(M)$. Then there exist $g \in \mathcal{U}(f)$ and $\delta>0$ such that non-hyperbolic periodic point $p_{g} \in P(g)$ and $p_{g}$ has a $\delta /$ 2-weak eigenvalue. By Remark 2.6, $f$ has a $\delta$-weak eigenvalue, which is a contradiction, by Lemma 2.8 .

From the above Proposition 2.4, we get the following results.

Proposition 2.9 Let $f \in \mathcal{G}^{\prime \prime}$. If $f$ has the usual limit shadowing property on a locally maximal homoclinic class $H_{f}(p)$ then there exist constants $m>0$ and $0<\lambda<1$ such that for any periodic point $q \in H_{f}(p)$,

$$
\begin{aligned}
& \prod_{i=0}^{\pi(q)-1} \|\left. D f^{m}\right|_{E^{s}\left(f^{i m}(q)\right)}||<\lambda^{\pi(q)}, \\
& \prod_{i=0}^{\pi(q)-1} \|\left. D f^{-m}\right|_{E^{u}\left(f^{-i m}(q)\right)}||<\lambda^{\pi(q)}
\end{aligned}
$$

and

$$
\left.\left.|| D f^{m}\right|_{E^{s}(q)}|| \cdot|| D f^{-m}\right|_{E^{u}\left(f^{-m}(q)\right)}||<\lambda^{2},
$$

where $\pi(q)$ denotes the period of $q$.

Let us recall Mañé's ergodic closing lemma in [10]. For any $\epsilon>0$, let $B_{\epsilon}(f, x)$ an $\epsilon$-tubular neighborhood of $f$-orbit of $x$, i.e.,

$$
B_{\varepsilon}(f, x)=\left\{y \in M: d\left(f^{n}(x), y\right)<\varepsilon, \text { for some } n \in \mathbb{Z}\right\} .
$$

Let $\Sigma_{f}$ be the set of points $x \in M$ such that for any $C^{1}$-neighborhood $\mathcal{U}(f)$ of $f$ and $\epsilon$ $>0$, there are $g \in \mathcal{U}(f)$ and $y \in P(g)$ satisfying $g=f$ on $M \backslash B_{\epsilon}(f, x)$ and $d\left(\dot{f}(x), g^{i}(y)\right)$ 
$\leq \epsilon$ for $0 \leq i \leq \pi(y)$. We say that a point $x \in M$ is well closable for $f \in \operatorname{Diff}(M)$, if for any $\epsilon>0$ there are $g \operatorname{Diff}(M)$ with $d_{1}(f, g)<\epsilon$ such that $d\left(f^{n}(x), g^{n}(p)\right)<\epsilon$ for any $0 \leq n \leq$ $\pi(p)$, where $\pi(p)$ is the period of $p$, and $d_{1}$ is the $C^{1}$-metric. Let $\Sigma_{f}$ denote the set of well closable points of $f$. In [10], Mañé showed that for any $f$-invariant Borel probability measure $\mu$ on $M, \mu\left(\Sigma_{f}\right)=1$. Let $\mathcal{M}$ be the space of all Borel measures $\mu$ on $M$ with the weak* topology. Then we know that for any ergodic measure $\mu \in \mathcal{M}$ of $f, \mu$ is supported on a periodic orbit $\mathcal{O}_{f}(p)=\left\{p, f(p), \ldots, f^{\pi(p)-1}(p)\right\}$ if and only if

$$
\mu=\frac{1}{\pi(p)} \sum_{i=0}^{\pi(p)-1} \delta_{f^{i}}(p)
$$

where $\delta_{x}$ is the atomic measure respecting $x$. In this regards, we mention some involving results. In $[11,12]$, Crovisier explained Mañé's ergodic closing lemma which gives the measure theoretical viewpoint on the approximation by periodic orbit, that is, any ergodic invariant probability measure $\mu$ of $C^{1}$-generic Diffeomorphism is the limit of a sequence of invariant measures supported by periodic orbits $\mathcal{O}_{f}\left(p_{n}\right)$. Moreover, the orbits $\mathcal{O}_{f}\left(p_{n}\right)$ converge to the support of $\mu$ for the Hausdorff topology. From the above facts, we get the following results.

Lemma 2.10 [11, Theorem 3.1] Let $H_{f}(p)$ be the homoclinic class of $p$. Then there is a measure $\mu \in \mathcal{M}_{f}\left(H_{f}(p)\right)$ which has $\sup p(\mu)=H_{f}(p)$, where $\mathcal{M}_{f}(\Lambda)=\{\mu$ : $\mu$ is an $f$ invariant Borel probability on $M$ such that $\operatorname{supp}(\mu) \subset \Lambda\}$, endowed with the weak" topology.

The following lemma is proved by Lee and Wen [5].

Lemma 2.11 [5, Lemma 2.3] There is a residual set $\mathcal{G}_{5} \subset \operatorname{Diff}(M)$ such that for any $f \in \mathcal{G}_{5}, f$ satisfies that any ergodic invariant measure $\mu$ of $f$ is the limit of sequence of ergodic invariant measure supported by periodic orbits $\mathcal{O}_{f}\left(p_{n}\right)$ in the weak topology. Moreover, the orbit $\mathcal{O}_{f}\left(p_{n}\right)$ converges to the support of $\mu$ in the Hausdor topology.

End of the proof of Theorem 1.1. Let $f \in \mathcal{G}_{6}=\mathcal{G}^{\prime \prime} \cap \mathcal{G}_{5}$, and let $q$ be a hyperbolic periodic point of $f$. Suppose that $f$ has the usual limit shadowing property on a locally maximal homoclinic class $H_{f}(p)$. From Propositions 2.1 and 2.4, we know that $H_{f}(p)$ admits a dominated splitting $T_{H_{f}(p)} M=E \oplus F$ with $\operatorname{dim}(E)=\operatorname{index}(p)$. To prove Theorem 1.1, it is enough to show that $D f^{n}$ is contracting on $E$ and $D f^{m}$ is expanding on $F$. To simplify, we denoted $f^{m}$ by $f$. By contradiction, we may assume that $D f$ is not contracting on $E$. Then we can find a point $x \in H_{f}(p)$ such that

$$
\left.\left.\prod_{i=0}^{n-1}|| D f\right|_{E\left(f^{i}(x)\right)}\right) \mid \geq 1,
$$

for $n \geq 0$. Define a probability measure

$$
\mu_{n}=\frac{1}{n} \sum_{i=0}^{n-1} \delta_{f^{i}(x)}
$$

where $\delta_{x}$ is the atomic measure respecting $x$. Then there exists $\mu_{n_{k}} \rightarrow \mu \in \mathcal{M}$ as $k$ $\rightarrow \infty$. We can see that $\mu$ is a $f$-invariant measure on $M$ and $\operatorname{supp}(\mu) \subset H_{f}(p)$. 
Then by Lemma 2.10, $\operatorname{supp}(\mu)=H_{f}(p)$. Thus

$$
\begin{aligned}
\left.\int \log || D f\right|_{E(x)} \| d \mu & =\left.\lim _{k \rightarrow \infty} \int \log || D f\right|_{E(x)}|| d \mu_{n_{k}} \\
& =\left.\lim _{k \rightarrow \infty} \int \frac{1}{n} \sum_{i=0} \log || D f\right|_{E\left(f^{i}(x)\right)} \| \geq 0 .
\end{aligned}
$$

By the ergodic decomposition theorem, there is an ergodic measure $v$ with $\operatorname{supp}(v)=$ $H_{f}(p)$ such that

$$
\left.\int \log || D f\right|_{E(x)}|| d v \geq 0
$$

Then by Lemma 2.11, we can choose a sequence of ergodic $f$-invariant measures $v_{n}$ such that the support of each $v_{n}$ is a periodic orbit $\mathcal{O}_{f}\left(p_{n}\right),\left\{v_{n}\right\}$ converges $v$ and $\mathcal{O}_{f}\left(p_{n}\right)$ converges to the support of $v$. Since $H_{f}(p)$ is locally maximal, we may assume that every $\mathcal{O}_{f}\left(p_{n}\right)$ is contained in $H_{f}(p)$ for sufficiently large $n$. By Proposition 2.4, we have

$$
\left.\int \log || D f\right|_{E(x)}|| d \nu_{n}<\log \lambda<0,
$$

for sufficiently large $n$. Since $v_{n} \rightarrow v$ in the weak topology, we see that

$$
\left.\int \log || D f\right|_{E(x)}\left\|d v_{n} \rightarrow \int \log \right\| D f-E(x) \| d \nu
$$

as $n \rightarrow \infty$. Thus we know

$$
\left.\int \log || D f\right|_{E(x)}|| d v<0 .
$$

This is a contradiction. Thus $D f^{m}$ is contracting on $E$. Similarly we can see that $D f^{m}$ is expanding on $F$.

$C^{1}$-generically, a locally maximal homoclinic class is locally maximal transitive set which is the following facts.

Lemma 2.12 [11, Theorem 4.10] For $C^{1}$-generic $f$, a locally maximal transitive set is a locally maximal homoclinic class $H_{V}(p)$, where $V$ is a compact neighborhood of $H_{f}$ $(p)$, and $H_{V}(p)$ is a locally maximal in $V$.

Corollary 2.13 For $C^{1}$-generic $f$, a locally maximal transitive set is hyperbolic.

\section{Acknowledgements}

The author wishes to express his deep appreciation to the referee for his careful reading of the manuscript. This work was supported by the Basic Science Research Program through the National Research Foundation of Korea (NRF) funded by the Ministry of Education, Science and Technology (No. 2011-0007649).

\section{Competing interests}

The author declares that they have no competing interests.

Received: 7 February 2012 Accepted: 27 June 2012 Published: 27 June 2012

\section{References}

. Pilyugin, SY: Shadowing in Dynamical Systems. In Lecture Notes in Math, vol. 1706,Springer Verlag, Berlin (1999) Abdenur, F, Diaz, LJ: Pseudo-orbit shadowing in the C'-topology. Discret Contin Dyn Syst. 2007, 223-245 (2007)

3. Eirola, T, Nevanlinna, O, Pilyugin, V: Limit shadowing property. Numer Funct Anal Optim. 18, 75-92 (1997). doi:10.1080/ 01630569708816748 
4. Pilyugin, SY: Sets of dynamical systems with various limit shadowing proper-ties. J Dyn Diff Equ. 19, 747-775 (2007). doi:10.1007/s10884-007-9073-2

5. Lee, K, Wen, X: Shadowable chain transitive sets of $C^{1}$-generic Diffeomorphisms. Bull Korean Math Soc. 49, 263-270 (2012). doi:10.4134/BKMS.2012.49.2.263

6. Hayashi, S: Diffeomorphisms in satisfy Axiom A. Ergodic Theory Dyn Syst. 12, 233-253 (1992)

7. Lee, M: Robustly transitive sets with generic Diffeomorphisms. (in press)

8. Yang, D, Gan, S: Expansive homoclinic classes. Nonlinearity. 22, 729-733 (2009). doi:10.1088/0951-7715/22/4/002

9. Sakai, K, Sumi, N, Yamamoto, K: Diffeomorphisms satisfying the specification property. Proc Am Math Soc. 138, 315-321 (2009)

10. Mãné, R: An ergodic closing lemma. Ann Math. 116, 503-540 (1982). doi:10.2307/2007021

11. Abdebur, F, Bonatti, C, Crovisier, S: Nonuniform hyperbolicity for $C^{1}$-generic diffeomorpgisms. Isr J Math. 183, 1-60 (2011). doi:10.1007/s11856-011-0041-5

12. Crovisier, S: Periodic orbits and chain transitive sets of $C^{1}$ Diffeomorphisms. Publ Math de L'iheś. 104, 87-141 (2006). doi:10.1007/s10240-006-0002-4

doi:10.1186/1687-1847-2012-91

Cite this article as: Lee: Usual limit shadowable homoclinic classes of generic diffeomorphisms. Advances in Difference Equations 2012 2012:91.

\section{Submit your manuscript to a SpringerOpen ${ }^{\odot}$} journal and benefit from:

- Convenient online submission

- Rigorous peer review

- Immediate publication on acceptance

- Open access: articles freely available online

- High visibility within the field

- Retaining the copyright to your article

Submit your next manuscript at $\gg$ springeropen.com 